\title{
Dengue infection with multiorgan dysfunction:-sofa score, arterial lactate and serum albumin levels are predictors of outcome
}

\author{
S Jog ${ }^{1 *}$, S Prayag ${ }^{2}$, P Rajhans ${ }^{3}$, K Zirpe ${ }^{4}$, S Dixit ${ }^{5}$, L Pillai ${ }^{6}$, J Shah ${ }^{7}$, M Penurkar ${ }^{8}$, A Kakrani ${ }^{9}$, A Yadav ${ }^{10}$, K Kadapatti $^{11}$ \\ , B Pawar ${ }^{12}$, P Joshi $^{13}$, D Salunke ${ }^{13}$, A Deshpande $^{14}$, D Patel $^{3}$, ISCCM Pune Research Initiative ( IPRI)
}

From ESICM LIVES 2015

Berlin, Germany. 3-7 October 2015

\section{Introduction}

Mortality in severe dengue infections is attributed to development of multiple organ dysfunctions. The manifestations of severe dengue are varied and unfortunately the exact morbidity and mortality in terms of organ dysfunction are not well studied in Indian context. We did a prospective multicentre observational study in fourteen tertiary care intensive care units of Pune city during July 2012 to December 2013. The aim was to estimate mortality in severe dengue infections with multiorgan organ dysfunction.

\section{Methods}

Newly admitted patients in the Intensive Care Units with history, clinical assessment and initial laboratory investigations suggestive / confirming diagnosis of Dengue Infection and having multiorgan dysfunction on SOFA (Sequential organ failure assessment) score (Severe Dengue) were screened for the enrollment in the study. Screening period was defined as first 24 hours within intensive care admission. Inclusion criteria were: Adults $>18$ years of age, confirmed diagnosis of Dengue Infection by IgM or NS- 1 antigen or Dengue PCR positive and at least two documented Organ Dysfunction by SOFA criteria. Exclusion Criteria were: ICU admission or study enrollment after 5 days of first documented multi organ dysfunction, Pregnancy or Confounding diagnosis (concurrent Malaria /Leptospira /Ricketssia/ Bacterial sepsis). Data was collected on demographics, clinical and laboratory variables. Data on SOFA scores was collected on day 1 and day 2 of enrollment into study.

${ }^{1}$ Deenanath Mangeshkar Hospital, Intensive Care Medicine, Pune, India Full list of author information is available at the end of the article

\section{Results}

A total of 113 patients met all inclusion and exclusion criteria. $30(26.55 \%)$ patients did not survive and remaining $83(73.45 \%)$ patients survived. Median time to death amongst those who did not survive was 5 (1.5-9) days. Univariable and multivariable Cox Proportional Hazard Risk model created using baseline data on SOFA score, $\mathrm{pH}$, need for external respiratory support, serum albumin level and highest arterial lactate to predict mortality revealed that worse SOFA score at baseline, higher arterial lactate at baseline and low serum albumin levels as significant predictors.

Every $\mathrm{mmol} / \mathrm{L}$ increase in arterial lactates, the risk of mortality increases by a factor of 1.27 and similarly for every 1 unit increase in SOFA score at baseline, mortality increased by a factor of 1.23 Thus presence of organ dysfunction and especially cardiovascular organ dysfunction as evident by high arterial lactates is associated with higher risk of mortality. Analysis of Delta SOFA also revealed worsening SOFA score during intensive care unit stay (SOFA score on day 1 versus SOFA score on day 2 was associated with increasing mortality.

\section{Conclusions}

Our study showed significantly high mortality in patients with severe Dengue Infection especially those with non hematological organ dysfunction. Our study also showed strong correlation of SOFA score and Delta SOFA score, low serum albumin and high arterial lactate with prediction of mortality.

\section{Funding}

ISCCM Pune Research Initiative 
Table 1

\begin{tabular}{|c|c|c|c|c|c|c|}
\hline & $\begin{array}{l}\text { Survivors ( } \mathrm{n} \\
=83 \text { ) }\end{array}$ & $\begin{array}{l}\text { Non-survivors ( } \mathrm{n} \\
=30 \text { ) }\end{array}$ & $\begin{array}{l}\text { Univariable Hazards ratio } \\
(95 \% \text { C.I.)) }\end{array}$ & $\begin{array}{l}\mathrm{p}- \\
\text { value }\end{array}$ & $\begin{array}{l}\text { Multivariable Hazards ratio } \\
\text { (95\% C.I.) }\end{array}$ & $\begin{array}{l}\mathrm{p}- \\
\text { value }\end{array}$ \\
\hline $\begin{array}{l}\text { Highest Arterial lactate (mmol/L) } \\
\text { Median (IQR) }\end{array}$ & $1.7(1.2-2.8)$ & $7.7(3.6-13.7)$ & $1.26(1.16-1.37)$ & $\begin{array}{c}< \\
0.001\end{array}$ & $1.27(1.13-1.43)$ & $\begin{array}{c}< \\
0.001\end{array}$ \\
\hline $\begin{array}{l}\text { Highest } \mathrm{pH} \text { at baseline }>7.35 \leq \\
7.35\end{array}$ & $\begin{array}{l}54(92 \%) 5 \\
(8 \%)\end{array}$ & $14(47 \%) 16(53 \%$ & $4.80(2.29-10.04)$ & $\begin{array}{c}< \\
0.001\end{array}$ & $0.85(0.23-3.17)$ & 0.81 \\
\hline Mechanical ventilation No Yes & $\begin{array}{l}47(60 \%) 31 \\
(40 \%)\end{array}$ & $6(20 \%) 24(80 \%)$ & $3.44(1.40-8.43)$ & $\begin{array}{c}< \\
0.007 \\
\end{array}$ & $0.25(0.06-1.09$ & 0.07 \\
\hline Serum Albumin $(\mathrm{gm} / \mathrm{dL})>3 \leq 3$ & $\begin{array}{l}54(67 \%) 27 \\
(33 \%)\end{array}$ & $7(23 \%) 23(77 \%)$ & $3.56(1.51-8.39)$ & $\begin{array}{l}< \\
0.004\end{array}$ & $0.30(0.09-0.97)$ & 0.045 \\
\hline $\begin{array}{l}\text { SOFA score on Day } 1 \text { Median } \\
\text { (IQR) }\end{array}$ & $5(4-7)$ & $11(10-14)$ & $1.25(1.13-1.39)$ & $\begin{array}{c}< \\
0.001\end{array}$ & $1.23(1.00-1.51)$ & 0.05 \\
\hline
\end{tabular}

British Journal of Haematology

Table 2

\begin{tabular}{lllllll}
\hline & Alive & Died & Univariable HR 95\% C.I. & p value & Multivariable HR 95 \% C.I. & p value \\
\hline SOFA Day 1 > SOFA Day 2 & $77(93 \%)$ & $3(10 \%)$ & $19.05(5.75-63.11)$ & $<0.001$ & $14.85(1.57-140.80)$ & 0.01 \\
\hline SOFA Day 1 < SOFA Day 2 & $6(7 \%)$ & $27(90 \%)$ & $19.05(5.75-63.11)$ & $<0.001$ & $14.85(1.57-140.80)$ & 0.01 \\
\hline
\end{tabular}

[Analysis of Delta SOFA]

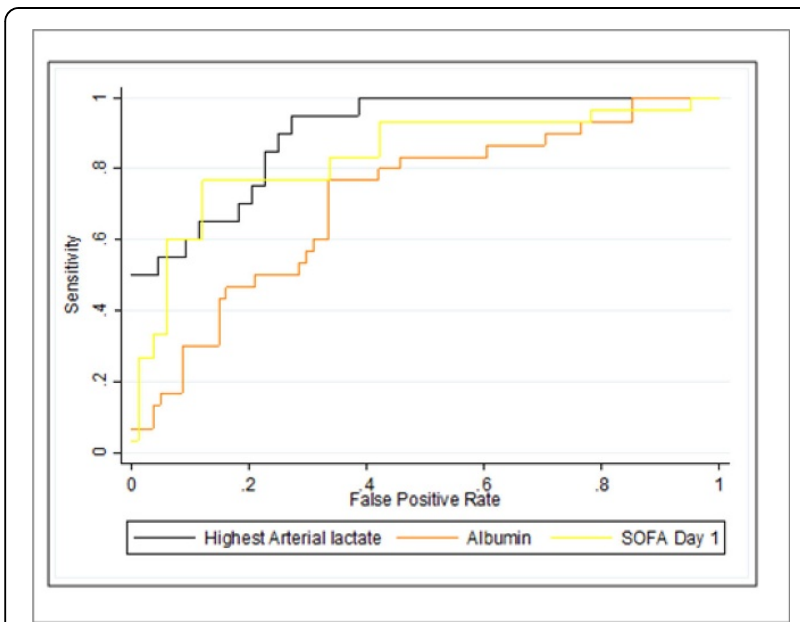

Figure 1 ROC curve for mortality.

\section{Authors' details}

${ }^{1}$ Deenanath Mangeshkar Hospital, Intensive Care Medicine, Pune, India.

${ }^{2}$ Shree Medical Foundation, Pune, India. ${ }^{3}$ Deenanath Mangeshkar Hospital, Pune, India. ${ }^{4}$ Ruby Hall Clinic, Pune, India. ${ }^{5}$ Sanjeevan Hospital, Pune, India. ${ }^{6}$ Sant Dnyaneshwar Hospital, Pune, India. ${ }^{7}$ Bharati Veedyapeeth Hospital, Pune, India. ${ }^{8}$ Shashwat Group of Hospitals, Pune, India. ${ }^{9}$ D.Y. Patil Hospital, Pune, India. ${ }^{10}$ Niramay Hospital, Pune, India. ${ }^{11}$ Jehangir Hospital, Pune, India. ${ }^{12}$ Mai Mangeshkar Hospital, Pune, India. ${ }^{13}$ ISCCM Pune Branch, Pune, India.

${ }^{14}$ John Hunter Children's Hospital, Newcastle, Newcastle, Australia.

Published: 1 October 2015

doi:10.1186/2197-425X-3-S1-A830

Cite this article as: Jog et al:: Dengue infection with multiorgan dysfunction:-sofa score, arterial lactate and serum albumin levels are predictors of outcome. Intensive Care Medicine Experimental 2015 3(Suppl 1):A830.

\section{Submit your manuscript to a SpringerOpen ${ }^{\circ}$ journal and benefit from:}

- Convenient online submission

- Rigorous peer review

- Immediate publication on acceptance

- Open access: articles freely available online

- High visibility within the field

- Retaining the copyright to your article

Submit your next manuscript at $>$ springeropen.com 